\title{
Impact of motion correction on reproducibility and spatial variability of quantitative myocardial $\mathrm{T}_{2}$ mapping
}

Sébastien Roujol ${ }^{1}$, Tamer A. Basha ${ }^{1,2}$, Sebastian Weingärtner ${ }^{1,3}$, Mehmet Akçakaya $^{1}$, Sophie Berg ${ }^{1}$, Warren J. Manning ${ }^{1,4}$ and Reza Nezafat ${ }^{{ }^{*}}$

\begin{abstract}
Background: To evaluate and quantify the impact of a novel image-based motion correction technique in myocardial $\mathrm{T}_{2}$ mapping in terms of measurement reproducibility and spatial variability.

Methods: Twelve healthy adult subjects were imaged using breath-hold (BH), free breathing (FB), and free breathing with respiratory navigator gating ( $F B+N A V$ ) myocardial $T_{2}$ mapping sequences. Fifty patients referred for clinical CMR were imaged using the FB + NAV sequence. All sequences used a $T_{2}$ prepared $\left(T_{2} p r e p\right)$ steady-state free precession acquisition. In-plane myocardial motion was corrected using an adaptive registration of varying contrast-weighted images for improved tissue characterization (ARCTIC). DICE similarity coefficient (DSC) and myocardial boundary errors (MBE) were measured to quantify the motion estimation accuracy in healthy subjects. $T_{2}$ mapping reproducibility and spatial variability were evaluated in healthy subjects using 5 repetitions of the FB + NAV sequence with either 4 or 20 $\mathrm{T}_{2}$ prep echo times (TE). Subjective $T_{2}$ map quality was assessed in patients by an experienced reader using a 4-point scale (1-non diagnostic, 4-excellent).

Results: ARCTIC led to increased DSC in BH data ( $0.85 \pm 0.08$ vs. $0.90 \pm 0.02, p=0.007)$, FB data $(0.78 \pm 0.13$ vs. $0.90 \pm$ $0.21, p<0.001)$, and FB + NAV data ( $0.86 \pm 0.05$ vs. $0.90 \pm 0.02, p=0.002)$, and reduced MBE in BH data $(0.90 \pm 0.40$ vs. $0.64 \pm 0.19 \mathrm{~mm}, p=0.005)$, FB data (1.21 \pm 0.65 vs. $0.63 \pm 0.10 \mathrm{~mm}, p<0.001)$, and FB + NAV data (0.81 \pm 0.21 vs. $0.63 \pm$ $0.08 \mathrm{~mm}, p<0.001$ ). Improved reproducibility (4TE: $5.3 \pm 2.5 \mathrm{~ms}$ vs. $4.0 \pm 1.5 \mathrm{~ms}, p=0.016 ; 20 \mathrm{TE}: 3.9 \pm 2.3 \mathrm{~ms}$ vs. $2.2 \pm$ $0.5 \mathrm{~ms}, p=0.002$ ), reduced spatial variability (4TE: $12.8 \pm 3.5 \mathrm{~ms}$ vs. $10.3 \pm 2.5 \mathrm{~ms}, p<0.001 ; 20 \mathrm{TE}: 9.7 \pm 3.5 \mathrm{~ms}$ vs. $7.5 \pm$ $1.4 \mathrm{~ms}$ ) and improved subjective score of $\mathrm{T}_{2}$ map quality (3.43 \pm 0.79 vs. $3.69 \pm 0.55, p<0.001$ ) were obtained using ARCTIC.
\end{abstract}

Conclusions: The ARCTIC technique substantially reduces spatial mis-alignment among $T_{2}$-weighted images and improves the reproducibility and spatial variability of in-vivo $T_{2}$ mapping.

Keywords: Motion correction, Image registration, Quantitative myocardial tissue characterization, Myocardial $T_{2}$ mapping

\footnotetext{
* Correspondence: rnezafat@bidmc.harvard.edu

'Department of Medicine (Cardiovascular Division), Beth Israel Deaconess

Medical Center, 330 Brookline Ave, Boston, MA 02215, USA

Full list of author information is available at the end of the article
} 


\section{Background}

The $\mathrm{T}_{2}$ relaxation time is dependent on the amount of free water [1] and can be exploited as a potential marker of inflammation and edema [2-7]. In cardiac MR $(\mathrm{CMR}), \mathrm{T}_{2}$ changes are generally assessed using a dark blood $\mathrm{T}_{2}$-weighted acquisition [8]. Elevated signal intensity in $\mathrm{T}_{2}$-weighted images have been reported in presence of several cardiomyopathies such as myocarditis [2, 3], Tako-Tsubo [4], and acute myocardial infarction [5-7]. However, this technique only provides qualitative measurements and image interpretation can be limited by several factors including regional signal variations induced by phased array coil, elevated signal induced by sub-endocardial stagnant blood, and signal loss caused by throughplane motion $[9,10]$.

Quantitative myocardial $\mathrm{T}_{2}$ mapping $[11,12]$ is an alternative technique, which shows promise for reducing uncertainties in interpretations of dark blood $\mathrm{T}_{2^{-}}$ weighted images. In this technique, several $\mathrm{T}_{2}$-weighted images are acquired, each with a different $\mathrm{T}_{2}$ contrast. The signal intensity obtained from the $\mathrm{T}_{2}$-weighted images is then fit to a physical model of $\mathrm{T}_{2}$ signal decay on a per-pixel basis, leading to the creation of a $T_{2}$ map. The acquisition of each $\mathrm{T}_{2}$-weighted image was initially performed using either spin echo/fast spin echo acquisitions [11-14] with varying echo times (TE) which results in very long scan time. Recently, $\mathrm{T}_{2}$-prepared ( $\left.\mathrm{T}_{2} \mathrm{prep}\right)$ [15] steady-state free precession (SSFP) acquisitions have been proposed and provide higher imaging efficiency [16]. These sequences can be acquired within a breathhold $[16,17]$ or under free breathing conditions with respiratory motion correction techniques $[16,18,19]$.

Despite the promise of this technique, its in-vivo reproducibility and precision have not been fully characterized. These two factors play a major role for clinical acceptance of any quantitative myocardial tissue characterization technique $[20,21]$. The presence of motion among $\mathrm{T}_{2^{-}}$ weighted images is one of the main challenges in $\mathrm{T}_{2}$ mapping and is expected to have important impact on the technique precision and reproducibility.

Breath-hold acquisitions can be used to reduce the impact of respiratory motion. However, some motion can still be detected in 40-60 \% of patients due to their limited breath-holding capabilities, as reported by several $\mathrm{T}_{1}$ mapping studies using breath-held acquisitions of 11-17 heart beats [22-25]. The breath-hold approach imposes severe time limitations on the number of acquired $\mathrm{T}_{2}$-weighted images (typically $\sim 3-4$ ) since a rest time of 4-6 heart beats is required between each acquisition to allow for full longitudinal magnetization recovery. Therefore, the use of a free breathing acquisition is attractive as it enables the acquisition of a larger number of $\mathrm{T}_{2}$-weighted images which may be beneficial to improve precision and reproducibility. On the other hand, free breathing acquisitions require the use of respiratory navigators to account for through plane motion and image registration algorithms to correct for residual inplane motion [18].

We recently developed a technique for Adaptive Registration of varying Contrast-weighted images for improved TIssue Characterization (ARCTIC) which we have evaluated for myocardial $\mathrm{T}_{1}$ mapping [23]. In this study, we sought to investigate the performance of ARCTIC for $\mathrm{T}_{2}$ mapping and its impact on in-vivo reproducibility and spatial variability of myocardial $\mathrm{T}_{2}$ estimates.

\section{Methods}

All subjects were scanned using a $1.5 \mathrm{~T}$ Philips Achieva (Philips Healthcare, Best, The Netherlands) scanner with a 32-channel cardiac phased array receiver coil. This study was health insurance portability and accountability act (HIPAA) compliant and the imaging protocol was approved by our institutional review board (Committee on Clinical Investigations (CCI)) at the Beth Israel Deaconess Medical Center. Written informed consent was obtained from each participant.

\section{$\mathrm{T}_{2}$ mapping acquisition scheme}

$\mathrm{T}_{2}$ mapping was performed using our recently reported $\mathrm{T}_{2}$ mapping sequence [26] in which multiple $\mathrm{T}_{2^{-}}$ weighted images are acquired using an electrocardiogram (ECG)-triggered $\mathrm{T}_{2}$ prep steady-state free precession (SSFP) acquisition with different $\mathrm{T}_{2}$ prep echo times $\left(\mathrm{TE}_{\mathrm{T} 2 \mathrm{P}}\right)$. A rest cycle of $6 \mathrm{~s}$ was used between the acquisitions of two successive $\mathrm{T}_{2}$-weighted images to ensure full re-growth of the longitudinal magnetization. The $\mathrm{TE}_{\mathrm{T} 2 \mathrm{P}}=0$ image was acquired using $90^{\circ}$ pulse followed immediately by a $-90^{\circ}$ pulse and a crusher gradient to ensure consistency with all other images in term of longitudinal signal reduction induced by imperfect $90^{\circ}$ and $-90^{\circ}$ flip angles used in the $\mathrm{T}_{2}$ prep. Finally, to model the signal re-growth induced by the SSFP imaging pulses, an infinitely long $\mathrm{T}_{2}$ prep echo time $\left(\mathrm{TE}_{\mathrm{T} 2 \mathrm{P}}=\infty\right)$ was simulated by acquiring an image immediately after a saturation pulse. In this study, this sequence has been evaluated with $4 \mathrm{~T}_{2}$ prep echo times ( $\mathrm{T}_{2 \mathrm{P}} 4 \mathrm{TE}: 0,25,50$, $\infty$ ) and $20 \mathrm{~T}_{2}$ prep echo times ( $\mathrm{T}_{2 \mathrm{P}} 20 \mathrm{TE}: 0,25,30,35$, $\ldots, 95,100, \infty, \infty, \infty)$. For free breathing acquisitions, a respiratory navigator positioned immediately prior to the $\mathrm{T}_{2}$ prep was used for end expiratory gating (window size $=5 \mathrm{~mm}$ ). No $\mathrm{T}_{2}$ prep or imaging pulses were applied if the navigator signal was outside the gating window to enable the acquisition of undisturbed signal in the next heartbeat.

\section{In-plane motion correction}

The ARCTIC approach was used to compensate for inplane motion between $\mathrm{T}_{2}$-weighted images [23]. In this 
approach, all images are registered individually to a common reference image, which was chosen as the first image of the series $\left(\mathrm{TE}_{\mathrm{T} 2 \mathrm{P}}=0\right)$. The motion was then estimated in a two-step process. Affine motion descriptors are first estimated over a region of interest surrounding the heart. This global transformation is then provided as input of a more sophisticated local non-rigid motion estimation step using an extended formulation of the optical flow problem which enables the simultaneous estimation of both motion field and intensity variations on a per-pixel basis [27]. An additional term is used to constrain the motion estimates based on prior automatic tracking of specific feature points in the images [28-30]. In this algorithm, both motion field and intensity variation map are solved using an iterative scheme. A multiresolution approach was used for the local non-rigid motion estimation step where the optical flow is initially estimated from first sub-resolution images and then refined using the full resolution images [31]. For each resolution level, the iterative scheme used 100 iterations and was repeated fifty times. These parameters were empirically optimized in this study. Since optical flow algorithms are well suitable for parallelization on graphic processing unit (GPU) [32-34], a GPU implementation of the method was used based on the compute unified device architecture (CUDA). More details about the algorithm can be found in [23].

\section{$T_{2}$ map reconstruction}

$\mathrm{T}_{2}$ maps were reconstructed offline using a 3-parameter curve fitting model.

$$
S\left(A, B, T_{2}, T_{n}\right)=A e^{-\mathrm{t}_{n} / T_{2}}+B .
$$

where $t_{n}$ is the $T_{2}$ prep echo time of $n^{\text {th }} T_{2}$-weighted image, and $\mathrm{A}, \mathrm{B}$, and $\mathrm{T}_{2}$ are the model parameters. $\mathrm{A}, \mathrm{B}$, and $T_{2}$ are estimated independently for each pixel using a Levenberg-Marquard optimizer with the online library provided in [35].

\section{In-vivo study in healthy subjects}

Twelve healthy adult subjects ( $32 \pm 16$ years, 6 male) without any history of cardiovascular disease underwent CMR examination. Each subject was imaged using eight $\mathrm{T}_{2}$ mapping sequences in the following order:

1. Breath-held $\mathrm{T}_{2 \mathrm{P}} 4 \mathrm{TE}$

2. Free breathing $\mathrm{T}_{2 \mathrm{P}} 4 \mathrm{TE}$ without respiratory navigator

3. Free breathing $\mathrm{T}_{2 \mathrm{P}} 4 \mathrm{TE}$ with respiratory navigator

4. Free breathing $\mathrm{T}_{2 \mathrm{P}} 20 \mathrm{TE}$ with respiratory navigator (5 repetitions).

All sequences were acquired in the short axis view using a single-shot ECG-triggered acquisition with SSFP imaging readout and the following parameters: field of view $=240 \times 240 \mathrm{~mm}^{2}$, in-plane resolution $=2.5 \times$ $2.5 \mathrm{~mm}^{2}$, slice thickness $=8 \mathrm{~mm}, \mathrm{TR} / \mathrm{TE}=2.7 \mathrm{~ms} /$ $1.35 \mathrm{~ms}$, flip angle $=85^{\circ}, 10$ linear ramp-up pulses, SENSE rate $=2$, acquisition window $=138 \mathrm{~ms}$, number of phase encoding lines $=51$, linear $\mathrm{k}$-space ordering. All $\mathrm{T}_{2}$ scans were acquired in the same short axis orientation at the mid-diastolic cardiac phase using one single mid-ventricular slice.

Accuracy of motion correction was evaluated in the first three scans (T2p4TE) by quantifying the motion between the $\mathrm{T}_{2}$-weighted images without (uncorrected) and with in-plane motion correction using ARCTIC (motion corrected). Endocardial and epicardial contours were manually drawn in all $\mathrm{T}_{2}$-weighted images of all $\mathrm{T}_{2}$ mapping scans. The two contours were used to create a binary representation of the myocardium for each $\mathrm{T}_{2^{-}}$ weighted image. The DICE similarity coefficient (DSC) [36] was then calculated between the myocardial binary mask of the reference image $\left(\mathrm{M}_{\mathrm{ref}}\right)$ and the myocardial binary mask of each $\mathrm{k}^{\text {th }} \mathrm{T}_{2}$-weighted image $\left(\mathrm{M}_{\mathrm{k}}\right)$ as follows:

$$
\mathrm{DSC}=\frac{2 \times \operatorname{area}\left(\mathrm{M}_{\mathrm{ref}} \cap \mathrm{M}_{\mathrm{k}}\right)}{\operatorname{area}\left(\mathrm{M}_{\mathrm{ref}}\right)+\operatorname{area}\left(\mathrm{M}_{\mathrm{k}}\right)}
$$

The myocardial boundary error (MBE), which provides a local alignment measure is also reported. MBE was measured as the average distance between the myocardial boundary of each $\mathrm{T}_{2}$-weighted image (boundary of $\mathrm{M}_{\mathrm{k}}$ ) and the myocardial boundary of the reference image (boundary of $M_{r e f}$ ) as follows:

$$
\operatorname{MBE}\left(M_{k}, M_{r e f}\right)=\frac{1}{N} \sum_{i=1}^{N}\left\|P_{M_{K}}^{i}-P_{M_{r e f}}^{\text {Closest }-i}\right\|_{2}
$$

Where $P_{M_{K}}^{i}$ is the $\mathrm{i}^{\text {th }}$ point along the boundary of $\mathrm{M}_{k}$, $P_{M_{r e f}}^{\text {Closest } i}$ is the closest point of $P_{M_{K}}^{i}$ located on the boundary of $M_{\text {ref }}$ Since $\mathrm{TE}_{\mathrm{T} 2 \mathrm{P}}=\infty$ images are very low signal-to-noise ratio (SNR) images, which makes the detection of the myocardial borders very difficult, no DSC/MBE were measured in those images. The statistical significant difference between DSCs (and MBEs) obtained with and without motion correction was evaluated using Wilcoxon signed rank tests. Statistical significance was considered at $p<0.05$.

The impact of in-plane motion correction on the reproducibility and spatial variability of $\mathrm{T}_{2}$ mapping was evaluated using the five $\mathrm{T}_{2 \mathrm{P}} 20 \mathrm{TE}$ scans. For each scan, $\mathrm{T}_{2}$ maps were reconstructed without (uncorrected) and with prior in-plane motion correction using ARCTIC (motion corrected). The endocardial and epicardial border of the myocardium and the insertion point were manually drawn on each $\mathrm{T}_{2}$ map. A six myocardial segment model [37] was automatically created for each single slice (1:anterior, 2:anterospetal, 3:inferospetal, 
4:inferior, 5:inferolateral, 6:anterolateral). Segment-based analysis of reproducibility and spatial variability of $T_{2}$ estimates was then performed. Spatial variability was defined as the standard deviation of $\mathrm{T}_{2}$ estimates over a given segment. Reproducibility was defined as the standard deviation over the 5 scans of the spatial average $T_{2}$ values in one given segment. Both reproducibility and spatial variability are reported in average over all segments for each subject, and in average over all subjects for each segment. To investigate the motion influence in $T_{2}$ mapping sequences using a limited number of $\mathrm{T}_{2}$ prep echo times, this overall analysis was repeated using a subset of the $T_{2}$-weighted images from each scan $\left(4 \mathrm{~T}_{2}\right.$ prep echo times of $\left.0,25,50, \infty\right)$. The statistical significant difference between uncorrected and ARCTIC motion corrected $\mathrm{T}_{2}$ reproducibility (and spatial variability) measured for each subject (in average over all myocardial segments) was evaluated using Wilcoxon signed rank tests.

\section{In-vivo study in patients}

Fifty patients referred for clinical CMR $(56 \pm 14$ y, 29 male) were imaged using the free breathing $\mathrm{T}_{2 \mathrm{P}} 4 \mathrm{TE} \mathrm{T}_{2}$ mapping sequence with respiratory navigator. All sequences were acquired in the short axis view using a single-shot ECG-triggered acquisition with SSFP imaging readout and the following parameters: field of view = $360 \times 360 \mathrm{~mm}^{2}$, in-plane resolution $=2 \times 2 \mathrm{~mm}^{2}$, slice thickness $=8 \mathrm{~mm}$, slice number $=3$, TR $/ \mathrm{TE}=2.9 \mathrm{~ms} /$ $1.45 \mathrm{~ms}$, flip angle $=85^{\circ}, 10$ linear ramp-up pulses, SENSE rate $=2$, acquisition window $=270 \mathrm{~ms}$, number of phase encoding lines $=93$, linear $\mathrm{k}$-space ordering. $\mathrm{T}_{2}$ maps were reconstructed without and with ARCTIC motion correction.

A subjective qualitative analysis was performed by an experienced cardiologist. The initial motion level in uncorrected data was assessed for each slice as "no motion", "small motion", or "large motion" by visual inspection of all uncorrected $\mathrm{T}_{2}$-weighted images. Subjective assessment of uncorrected and motion correction $\mathrm{T}_{2}$ maps (150 $\mathrm{T}_{2}$ maps) followed. Each pair of uncorrected and motion correction $\mathrm{T}_{2}$ maps were shown simultaneously to the reader side by side in a random order. The reader was blinded to the reconstruction approach (uncorrected vs. motion corrected). Each map was assessed in term of overall quality (1-non diagnostic/large artifacts/ no confidence in interpreting $\mathrm{T}_{2}$ values in more than half of the myocardial segments, 2-fair/moderate artifacts/confidence in interpreting $T_{2}$ values in more than half of the myocardial segments, 3-good/small motion artifacts/no confidence in interpreting $T_{2}$ values in at most one myocardial segment, 4-excellent/no motion artifact/confidence in interpreting $\mathrm{T}_{2}$ values in all myocardial segments). Furthermore, for each pair of $T_{2}$ maps, the reader was asked to evaluate if any of the two $\mathrm{T}_{2}$ map had "1-inferior", "2similar", or "3-superior" quality. Wilcoxon signed rank test was used to test the null hypothesis that the difference of overall $\mathrm{T}_{2}$ map quality scores between uncorrected and motion corrected $\mathrm{T}_{2}$ maps was zero. Statistical significance was considered at $\mathrm{p}<0.05$.

\section{Results}

All scans were successful. The nominal scan time (assuming 100\% gating efficiency) corresponded to 13 heart beats for the $\mathrm{T}_{2 \mathrm{P}} 4 \mathrm{TE}$ sequence and to 99 heart beats for the $\mathrm{T}_{2 \mathrm{P}} 20 \mathrm{TE}$ sequence. The employed ARCTIC motion correction and reconstruction of one $\mathrm{T}_{2}$ map with $20 \mathrm{~T}_{2}$ prep echo times was $20 \mathrm{~s}$.

Figure 1 shows an example of the remaining in-plane motion between $T_{2}$-weighted images acquired in one healthy subject using the $\mathrm{T}_{2 \mathrm{P}} 4 \mathrm{TE}$ sequence under breath-hold, free breathing, and free breathing with respiratory navigator gating. Motion artifacts can be observed in the reconstructed $\mathrm{T}_{2}$ maps (see white arrows). In-plane motion correction improves the spatial alignment of $\mathrm{T}_{2}$-weighted images and results in visually improved $\mathrm{T}_{2}$ map quality (Figure 1 ).

Figure 2 shows quantitative metrics of motion accuracy (DSC and MBE) obtained in healthy subjects using the three aforementioned acquisition sequences. Increased DSC and reduced MBE were observed in each of the three acquisition sequences. In the remaining part of this paragraph, DSC and MBE are reported as (uncorrected data vs. motion corrected data using ARCTIC). On average for all subjects, the DSC increased in breathhold data $(0.85 \pm 0.08$ vs. $0.90 \pm 0.02, p=0.007)$, free breathing data ( $0.78 \pm 0.13$ vs. $0.90 \pm 0.21, p<0.001)$, and free breathing data with respiratory navigator gating $(0.86 \pm 0.05$ vs. $0.90 \pm 0.02, p=0.002)$. The MBE decreased in breath-hold data $(0.90 \pm 0.40$ vs. $0.64 \pm$ $0.19 \mathrm{~mm}, p=0.005)$, free breathing data $(1.21 \pm 0.65$ vs. $0.63 \pm 0.10 \mathrm{~mm}, p<0.001)$, and free breathing data with respiratory navigator gating $(0.81 \pm 0.21$ vs. $0.63 \pm$ $0.08 \mathrm{~mm}, p<0.001$ ).

Figure 3 shows an example of multiple $\mathrm{T}_{2}$ maps obtained in one healthy subject using the $\mathrm{T}_{2 \mathrm{P}} 20 \mathrm{TE}$ sequence acquired under free breathing conditions with respiratory navigator gating. $T_{2}$ maps are shown when reconstructed from only $4 \mathrm{~T}_{2}$ prep echo times and from all $20 \mathrm{~T}_{2}$ prep echo times. The level of artifacts in uncorrected $\mathrm{T}_{2}$ maps appears higher than in motion corrected $\mathrm{T}_{2}$ maps (see white arrows). As expected, motion artifact patterns have high spatial variability in uncorrected $T_{2}$ maps. Furthermore, the spatial variability of the myocardial $\mathrm{T}_{2}$ estimates appears well reduced when using all 20 $\mathrm{T}_{2}$ prep echo times compared to only $4 \mathrm{~T}_{2}$ prep echo times. 


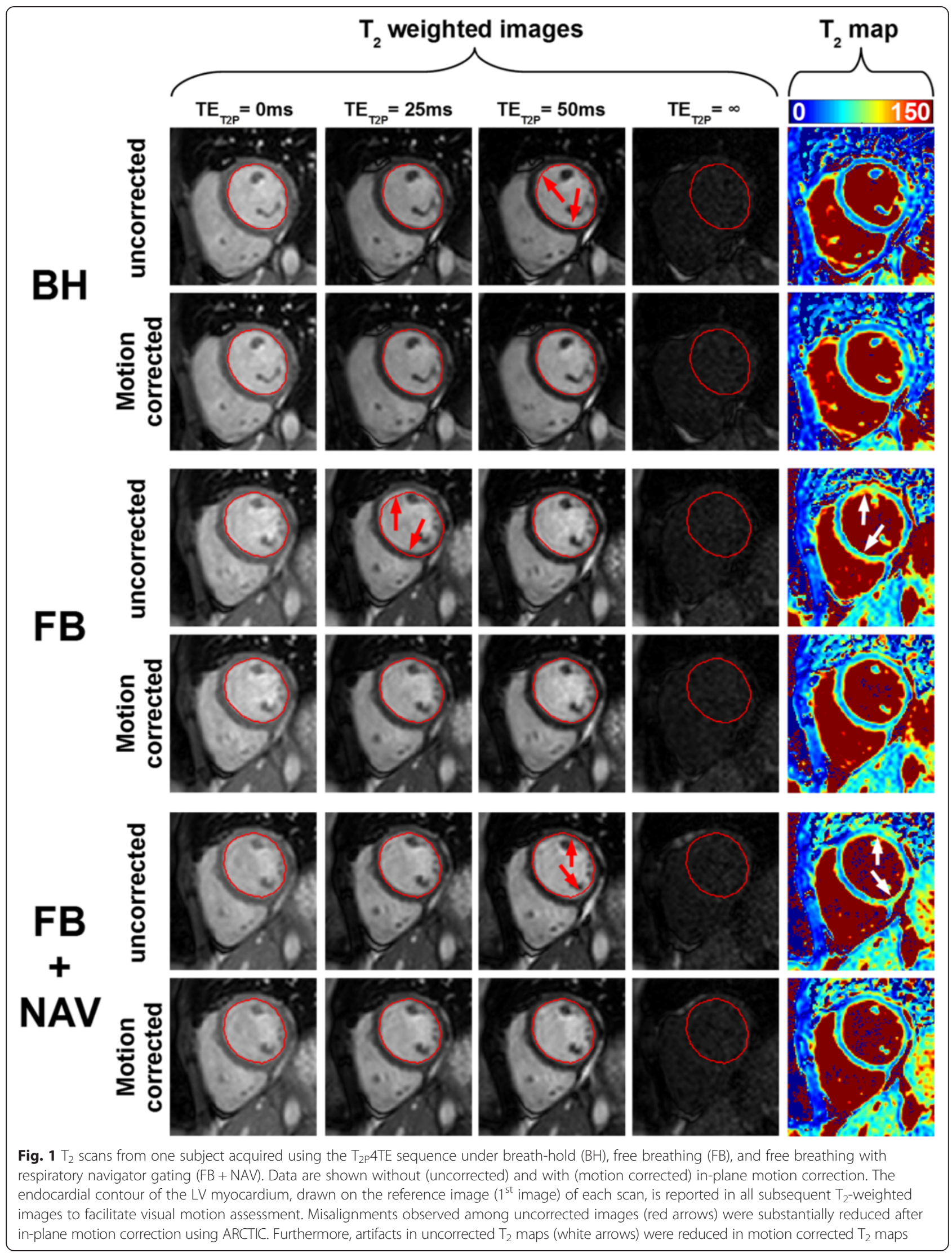



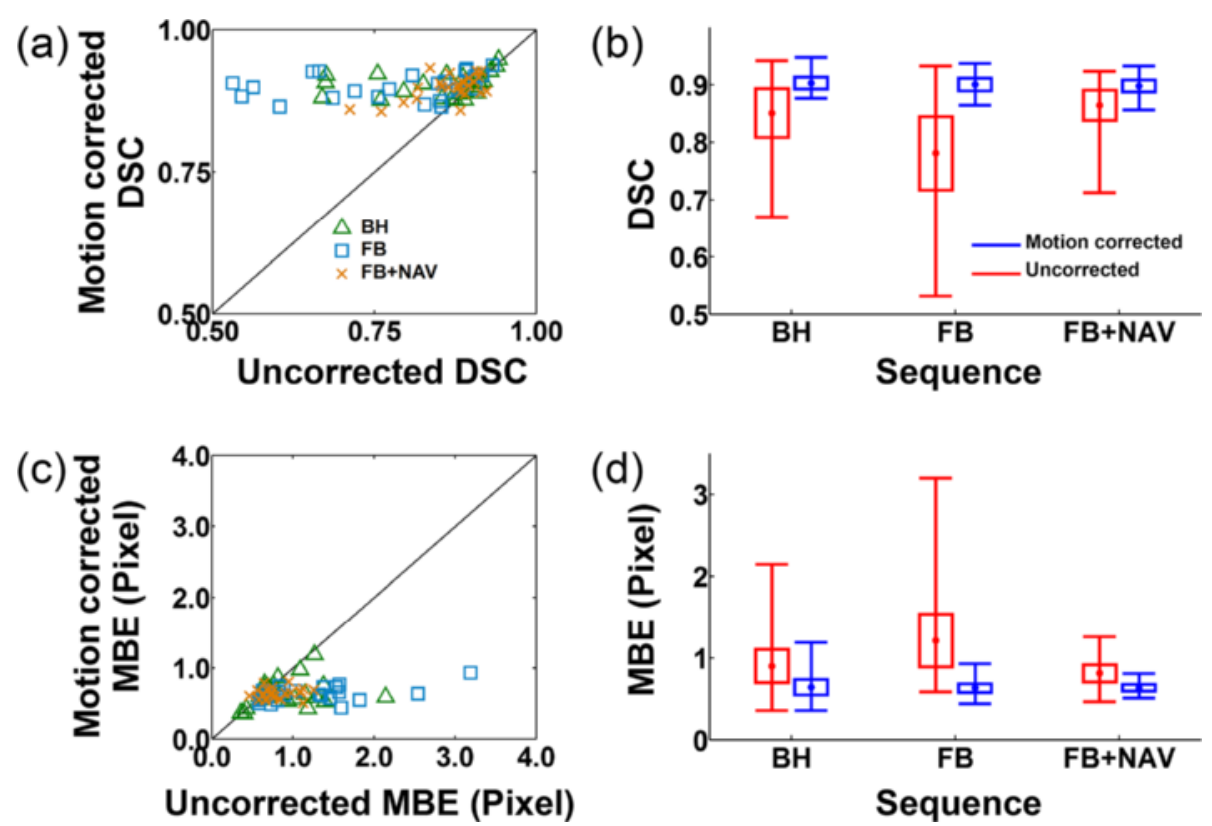

Fig. 2 Dice similarity coefficient (DSC) (a,b) and myocardial boundary error (MBE) (c,d) obtained using the $T_{2 p} 4 T E$ sequence under breath-hold $(\mathrm{BH})$, free breathing $(\mathrm{FB})$, and free breathing with respiratory navigator gating ( $F B+N A V$ ). DSCs and MBEs of all $\mathrm{T}_{2}$-weighted images are shown in (a) and (b), respectively. (b) and (d) show DSC and MBE as average (central dot), standard deviation (box size) and minimum/maximum (whiskers) over all subjects and all $T_{2}$-weighted images (except the $T_{2} p r e p=\infty$ images). In-plane motion correction improves the DSC and reduces the MBE for all cases. Furthermore, motion corrected DSC and MBE were similar for all 3 acquisitions (i.e. BH, FB, FB + NAV)

Figures 4 and 5 summarize the reproducibility and spatial variability of $\mathrm{T}_{2}$ measurements obtained in healthy subjects using the $\mathrm{T}_{2 \mathrm{P}} 20 \mathrm{TE}$ sequence. Results are shown for uncorrected and motion corrected $T_{2}$ maps reconstructed using either $4 \mathrm{~T}_{2}$ prep echo times or $20 \mathrm{~T}_{2}$ prep echo times. Reproducibility and spatial variability are reported as uncorrected $\mathrm{T}_{2}$ maps vs. motion corrected $\mathrm{T}_{2}$ maps using ARCTIC. Improved reproducibility was observed over all subjects and myocardial segments in $\mathrm{T}_{2}$ maps reconstructed from $4 \mathrm{~T}_{2}$ prep echo times $(5.3 \pm 2.5 \mathrm{~ms}$ vs. $4.0 \pm 1.5 \mathrm{~ms}, p=0.016)$ and 20 $\mathrm{T}_{2}$ prep echo times $(3.9 \pm 2.3 \mathrm{~ms}$ vs. $2.2 \pm 0.5 \mathrm{~ms}, p=$ $0.002)$. Similarly, reduced spatial variability was observed over all subjects and myocardial segments in $\mathrm{T}_{2}$ maps reconstructed from $4 \mathrm{~T}_{2}$ prep echo times $(12.8 \pm 3.5 \mathrm{~ms}$ vs. $10.3 \pm 2.5 \mathrm{~ms}, p<0.001)$ and $20 \mathrm{~T}_{2}$ prep echo times $(9.7 \pm 3.5 \mathrm{~ms}$ vs. $7.5 \pm 1.4 \mathrm{~ms}, p=0.005)$.

As expected, $\mathrm{T}_{2}$ maps reconstructed using $20 \mathrm{~T}_{2}$ prep echo times had better reproducibility than those reconstructed using only $4 \mathrm{~T}_{2}$ prep echo times in both uncorrected data $(3.9 \pm 2.3 \mathrm{~ms}$ vs. $5.3 \pm 2.5 \mathrm{~ms}$, respectively, $p=$ $0.007)$ and motion corrected $(2.2 \pm 0.5 \mathrm{~ms}$ vs. $4.0 \pm 1.5 \mathrm{~ms}$, respectively, $p<0.001)$. The spatial variability of myocardial $\mathrm{T}_{2}$ estimates reconstructed using $20 \mathrm{~T}_{2}$ prep echo times was also lower than the one obtained with $4 \mathrm{~T}_{2}$ prep echo times in both uncorrected data $(9.7 \pm 3.5 \mathrm{~ms}$ vs. $12.8 \pm$
$3.5 \mathrm{~ms}$, respectively, $p<0.001)$ and motion corrected data (7.5 $\pm 1.4 \mathrm{~ms}$ vs. $10.3 \pm 2.5 \mathrm{~ms}$, respectively, $p<0.001)$.

Figure 6 shows example uncorrected and ARCTIC motion corrected $T_{2}$ maps obtained in four patients. Large regional variations and artifacts can be observed in uncorrected $\mathrm{T}_{2}$ maps (see white arrows). The proposed ARCTIC motion correction substantially improved the $\mathrm{T}_{2}$ map quality in all 4 patients.

Figure 7 shows the subjective assessment of $T_{2}$ map quality obtained in 50 patients. Overall $(N=$ $150 \mathrm{~T}_{2}$ maps), ARCTIC motion corrected $\mathrm{T}_{2}$ maps had higher quality score than uncorrected $\mathrm{T}_{2}$ maps $(3.69 \pm 0.55$ vs. $3.43 \pm 0.79, p<0.001)$. In the relative comparison of $\mathrm{T}_{2}$ map quality, uncorrected $\mathrm{T}_{2}$ maps has superior, similar, and inferior quality than ARCTIC motion corrected $\mathrm{T}_{2}$ maps in 4 maps (3\%), 99 maps (66\%), and 47 maps (31\%), respectively. Furthermore, the motion level was assessed as "no motion" in 35 slices (23\%), "small motion" in 69 slices (46\%), and "large motion" in 46 slices (30\%). In "no motion" data, all ARCTIC motion corrected and uncorrected $\mathrm{T}_{2}$ maps received a subjective quality score of 4.0 and $97 \%$ of them had similar relative quality. In "small motion" data, ARCTIC motion corrected $\mathrm{T}_{2}$ maps had higher subjective quality score $(3.71 \pm 0.49$ vs. $3.61 \pm$ $0.60, p=0.015)$ and superior (23\%), similar (75\%) and 


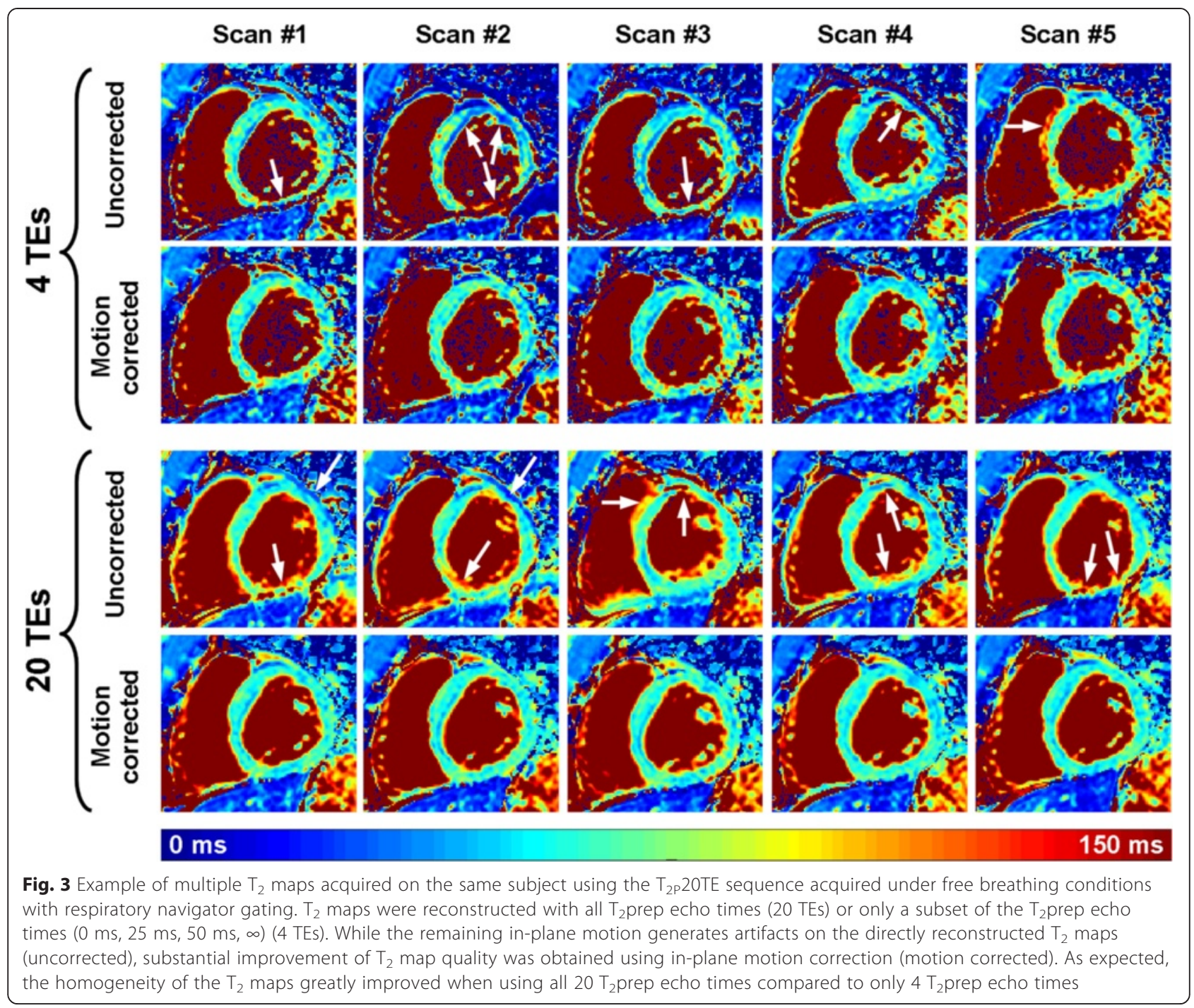

inferior (1\%) relative quality than uncorrected $\mathrm{T}_{2}$ maps. In "large motion" data, ARCTIC motion corrected $\mathrm{T}_{2}$ maps had higher subjective quality score $(3.41 \pm 0.69$ vs. $2.72 \pm$ $0.83, p<0.001)$ and superior $(65 \%)$, similar $(28 \%)$ and inferior $(6 \%)$ relative quality than uncorrected $\mathrm{T}_{2}$ maps.

\section{Discussion}

In this study, we demonstrate the benefit of in-vivo inplane ARCTIC motion correction in myocardial $T_{2}$ mapping. The method provides improved alignment of the myocardium in $\mathrm{T}_{2}$-weighted images acquired with breath-hold acquisitions and free breathing acquisitions with and without respiratory navigator gating. ARCTIC motion correction improves $\mathrm{T}_{2}$ map quality which results in improved reproducibility and spatial variability of myocardial $\mathrm{T}_{2}$ estimates. Finally, the CPU/GPU implementation of ARCTIC substantially reduces the computation time of the $T_{2}$ map reconstruction to $20 \mathrm{~s}$ which is suitable for clinical applicability.
DSCs and MBEs found in this study are in good agreement with previous studies [23-25, 38]. As expected higher mis-alignments were observed using free breathing acquisitions without respiratory navigator gating. DSCs/MBEs improvement was obtained in all three types of acquisitions. This confirms the benefit of motion correction, even for data acquired with a breathhold. This is likely because $40-60 \%$ of patients fail to sustain a stable breath-hold in these conditions [23-25]. Furthermore, similar DSCs/MBEs were obtained after motion correction using the three acquisition conditions (breath-hold and free breathing with and without respiratory navigator). It is important to note that through-plane motion cannot be compensated when using the free breathing acquisition without respiratory navigator gating. In this case, the efficacy of in-plane motion correction algorithms depends on the subject's heart orientation in relation to his respiratory movement. The use of respiratory navigator appears thus 


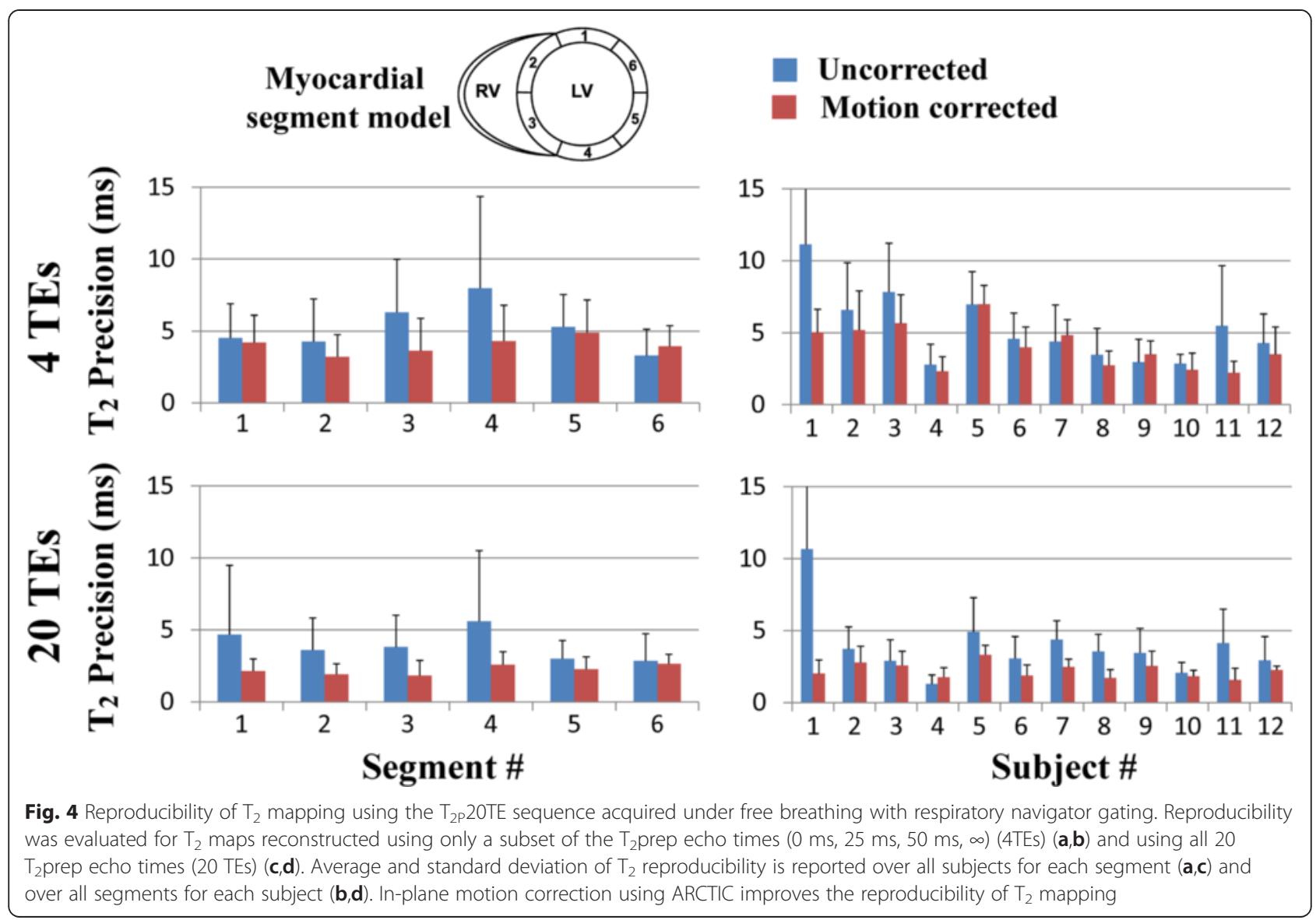

desirable to enable through plane motion compensation in free breathing acquisitions. The registration accuracy was not evaluated in the $\mathrm{TE}_{\mathrm{T} 2 \mathrm{P}}=\infty$ images since the contrast is too low to identify the myocardium. Motion correction is expected to have slightly lower accuracy in those images due to the expected limited ability to compensate for complex motion.

The ARCTIC approach successfully corrected the encountered motion in all subjects. In this study, the heart motion patterns were mainly influenced by the breathing activity of the subjects and to lesser extent to their RRinterval variations. However, the motion pattern can be more complex in patients imaged during arrhythmic events. The performance of the method in such conditions was not investigated and should be addressed in future work.

The reproducibility and spatial variability of $\mathrm{T}_{2}$ mapping was improved using ARCTIC. The use of $20 \mathrm{~T}_{2}$ weighted images improved the reproducibility and the spatial variability of $\mathrm{T}_{2}$ mapping (over the use of only $4 \mathrm{~T}_{2}$-weighted images) by a factor of 2 and 1.4, respectively. Therefore, the choice of the number of $\mathrm{T}_{2}$ prep echo times depends on the desired trade-off between acquisition time and $\mathrm{T}_{2}$ map quality. Further studies are warranted to determine the clinically relevant threshold providing satisfactory $T_{2}$ map quality in a reasonable amount of time.

Reproducibility and spatial variability of $\mathrm{T}_{2}$ estimates were found similar in all myocardial segments when using 20 TEs. However, slight differences seemed to be observed when using 4TEs only, especially in the myocardial segment \#4 (inferior wall). Several factors could have contributed to this observation including 1) increased sensitivity to cardiac motion and partial voluming in the free wall due to reduced wall thickness, 2) increased field inhomogeneity in myocardial segments located at the heart/lung interface. Future studies are warranted to study the impact of each of these factors.

In this study, the data were acquired using our recently developed $\mathrm{T}_{2}$ mapping sequence. The ARCTIC approach is expected to provide similar motion correction performance using other $\mathrm{T}_{2}$ mapping sequences. Nevertheless, the impact of motion correction on the reproducibility and spatial variability of other $T_{2}$ mapping sequences may be different and is beyond the scope of this study. Furthermore, all data were acquired in 2D. $3 \mathrm{D}$ myocardial $\mathrm{T}_{2}$ mapping may represent a valuable approach for true $3 \mathrm{D}$ assessment of pathological tissues $[39,40]$. The extension of the ARCTIC approach to 3D is straightforward and is expected to provide similar 

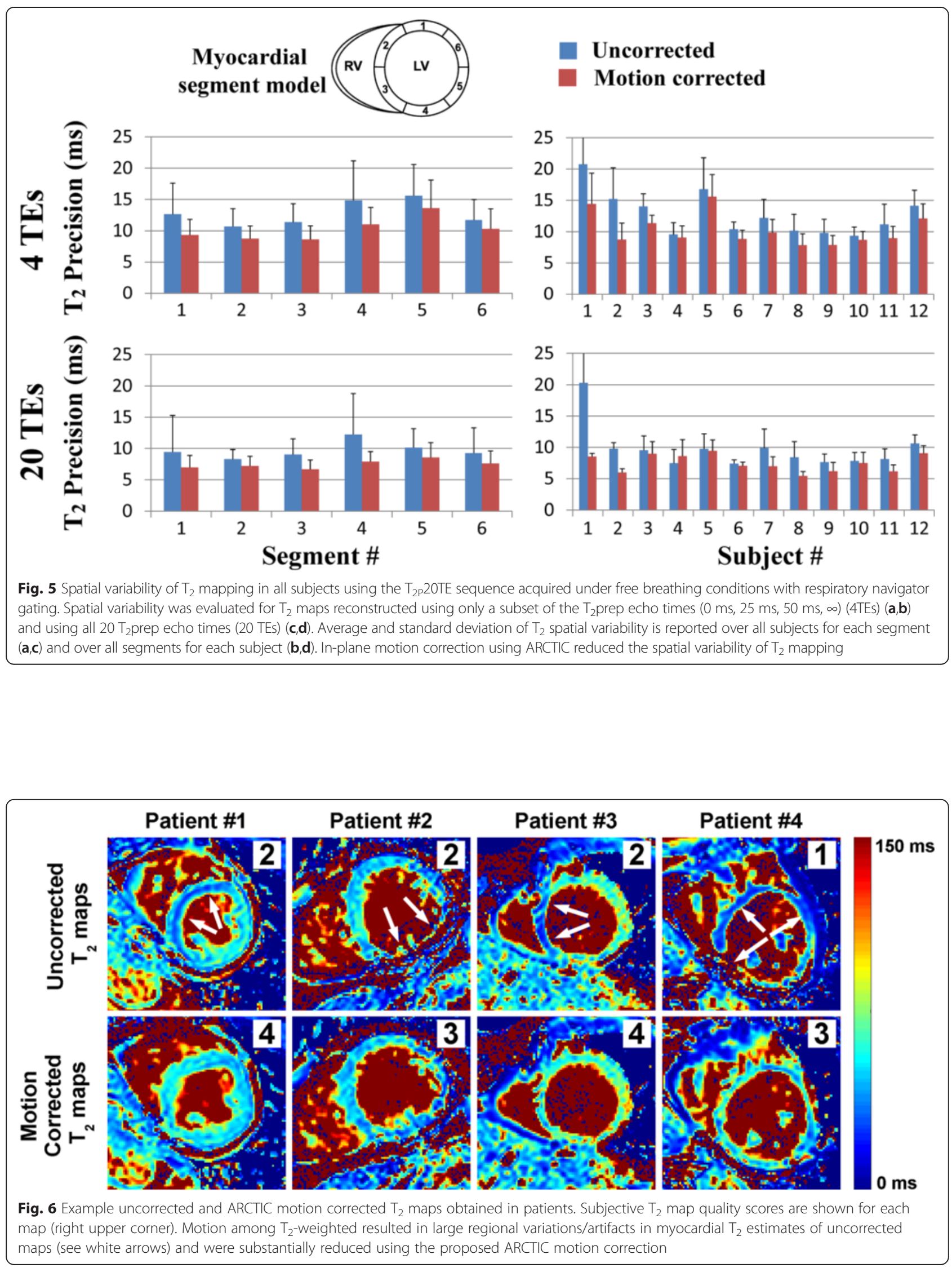

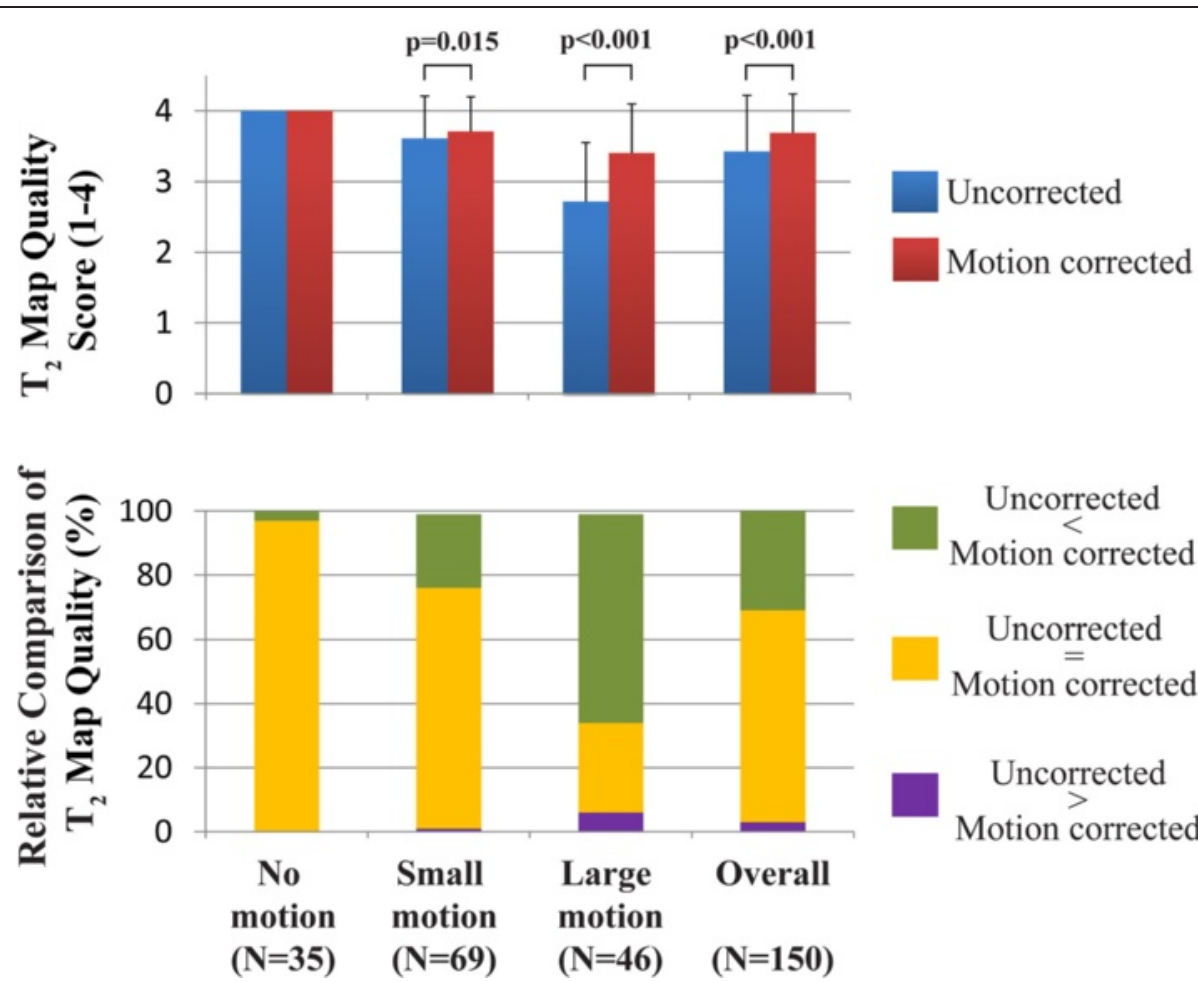

Uncorrected
$<$
Motion corrected

Uncorrected

\section{Uncorrected}

Motion corrected

$$
(\mathbf{N = 3 5 )} \quad(\mathbf{N}=69) \quad(\mathrm{N}=46) \quad(\mathrm{N}=150)
$$

Fig. 7 Subjective assessment of $T_{2}$ map quality in patients. In-plane motion correction using ARCTIC increased $T_{2}$ map quality scores (3.69 \pm 0.55 vs. $3.43 \pm 0.79, p<0.001$ )

improvement of the reproducibility and spatial variability of $3 \mathrm{D} \mathrm{T}_{2}$ mapping.

There are several limitations in this study. In the invivo analysis of reproducibility and spatial variability, the 4TEs data were extracted from the $\mathrm{T}_{2 \mathrm{P}} 20 \mathrm{TE}$ sequence and were thus not acquired using the $\mathrm{T}_{2 \mathrm{P}} 4 \mathrm{TE}$ sequence. However, since the $\mathrm{T}_{2 \mathrm{P}} 20 \mathrm{TE}$ sequence was acquiring with respiratory gating, the potential bias in reproducibility and spatial variability obtained in the 4TEs data should have been kept to the minimum. Finally, the study was only performed in healthy adult subjects with limited sample size. Further studies are warranted to confirm the benefit of the ARCTIC approach to improve the reproducibility and spatial variability of myocardial $\mathrm{T}_{2}$ mapping in patients.

\section{Conclusions}

The ARCTIC technique substantially reduces spatial mis-alignment among $\mathrm{T}_{2}$-weighted images. This method improves the reproducibility and reduces the spatial variability of in-vivo $T_{2}$ mapping. Furthermore, the invivo reproducibility and spatial variability of $\mathrm{T}_{2}$ mapping is improved using a higher number of $\mathrm{T}_{2}$ prep echo times combined with ARCTIC motion correction.

\begin{abstract}
Abbreviations
T2prep: T2 prepared; ARCTIC: Adaptive registration of varying contrast-weighted images for improved tissue characterization; BH: Breath-hold; FB: Free breathing; FBNAV: Free breathing conditions with respiratory navigator gating; DSC: DICE similarity coefficient; MBE: Myocardial boundary errors (MBE); TE: Eecho times; SSFP: Steady-state free precession; HIPAA: Health insurance portability and accountability act; ECG: Electrocardiogram; TET2P: T2prep echo times; TR: Repetition time; FOV: Field of view; SENSE: Sensitivity encoding; SNR: Signalto-noise ratio; GPU: Graphic processing unit; CUDA: Compute unified device architecture.
\end{abstract}

\section{Competing interests}

SR, WJM and RN have a pending patent for methods for correcting motion for tissue characterization sequences. TB, MA, WJM, and RN have a pending patent for system and method for assessing $T_{2}$ relaxation times with improved accuracy.

\section{Authors' contributions}

SR participated in the study design and coordination, developed the ARCTIC approach, carried out the motion correction/reconstruction of the data, performed the data analysis and drafted the manuscript. TB developed the prospective $T_{2}$ mapping sequence and participated in data acquisition. SW developed the $T_{2}$ mapping reconstruction code. MA participated in the data acquisition. SB was in charge of subject recruitment. WJM helped in revising the manuscript. RN conceived the study, participated in the study design and interpretation of the data. All authors read and approved the final manuscript.

\section{Acknowledgements}

The project described was partially supported by NIH R01EB008743-01A2, and Samsung Electronics, Suwon, South Korea. 


\section{Author details}

${ }^{1}$ Department of Medicine (Cardiovascular Division), Beth Israel Deaconess Medical Center, 330 Brookline Ave, Boston, MA 02215, USA. ${ }^{2}$ Biomedical Engineering Department, Cairo University, Giza, Egypt. ${ }^{3}$ Computer Assisted Clinical Medicine, University Medical Center Mannheim, Heidelberg University, Mannheim, Germany. ${ }^{4}$ Radiology, Beth Israel Deaconess Medical Center and Harvard Medical School, Boston, MA, USA

Received: 29 January 2015 Accepted: 1 May 2015

Published online: 12 June 2015

\section{References}

1. Higgins CB, Herfkens R, Lipton MJ, Sievers R, Sheldon P, Kaufman L, et al. Nuclear magnetic resonance imaging of acute myocardial infarction in dogs: alterations in magnetic relaxation times. Am J Cardiol. 1983;52(1):184-8.

2. Abdel-Aty H, Boye P, Zagrosek A, Wassmuth R, Kumar A, Messroghli D, et al. Diagnostic performance of cardiovascular magnetic resonance in patients with suspected acute myocarditis: comparison of different approaches. J Am Coll Cardiol. 2005;45(11):1815-22.

3. Friedrich MG, Sechtem U, Schulz-Menger J, Holmvang G, Alakija P, Cooper $\mathrm{LT}$, et al. Cardiovascular magnetic resonance in myocarditis: A JACC White Paper. J Am Coll Cardiol. 2009;53(17):1475-87.

4. Abdel-Aty H, Cocker M, Friedrich MG. Myocardial edema is a feature of Tako-Tsubo cardiomyopathy and is related to the severity of systolic dysfunction: insights from T2-weighted cardiovascular magnetic resonance. Int J Cardiol. 2009:132(2):291-3.

5. Abdel-Aty H, Zagrosek A, Schulz-Menger J, Taylor AJ, Messroghli D, Kumar A, et al. Delayed enhancement and T2-weighted cardiovascular magnetic resonance imaging differentiate acute from chronic myocardial infarction. Circulation. 2004;109(20):2411-6.

6. Raman SV, Simonetti OP, Winner 3rd MW, Dickerson JA, He X, Mazzaferri Jr EL, et al. Cardiac magnetic resonance with edema imaging identifies myocardium at risk and predicts worse outcome in patients with non-ST-segment elevation acute coronary syndrome. J Am Coll Cardiol. 2010;55(22):2480-8.

7. Cury RC, Shash K, Nagurney JT, Rosito G, Shapiro MD, Nomura CH, et al. Cardiac magnetic resonance with $\mathrm{T} 2$-weighted imaging improves detection of patients with acute coronary syndrome in the emergency department. Circulation. 2008;118(8):837-44.

8. Simonetti OP, Finn JP, White RD, Laub G, Henry DA. "Black blood" T2-weighted inversion-recovery MR imaging of the heart. Radiology. 1996;199(1):49-57.

9. Abdel-Aty $\mathrm{H}$, Simonetti $\mathrm{O}$, Friedrich MG. T2-weighted cardiovascular magnetic resonance imaging. J Magn Reson Imaging. 2007;26(3):452-9.

10. Arai AE. Using magnetic resonance imaging to characterize recent myocardial injury: utility in acute coronary syndrome and other clinical scenarios. Circulation. 2008;118(8):795-6.

11. Bottomley PA, Foster TH, Argersinger RE, Pfeifer LM. A review of normal tissue hydrogen NMR relaxation times and relaxation mechanisms from 1-100 MHz: dependence on tissue type, NMR frequency, temperature, species, excision, and age. Med Phys. 1984;11(4):425-48

12. McNamara MT, Higgins CB, Schechtmann N, Botvinick E, Lipton MJ, Chatterjee $\mathrm{K}$, et al. Detection and characterization of acute myocardial infarction in man with use of gated magnetic resonance. Circulation 1985;71(4):717-24

13. Foltz WD, Stainsby JA, Wright GA. T2 accuracy on a whole-body imager. Magn Reson Med. 1997;38(5):759-68.

14. He T, Gatehouse PD, Anderson LJ, Tanner M, Keegan J, Pennell DJ, et al. Development of a novel optimized breathhold technique for myocardial T2 measurement in thalassemia. J Magn Reson Imaging. 2006;24(3):580-5.

15. Brittain JH, Hu BS, Wright GA, Meyer CH, Macovski A, Nishimura DG. Coronary angiography with magnetization-prepared T2 contrast. Magn Reson Med. 1995:33(5):689-96.

16. Huang TY, Liu YJ, Stemmer A, Poncelet BP. T2 measurement of the human myocardium using a T2-prepared transient-state TrueFISP sequence. Magn Reson Med. 2007:57(5):960-6.

17. Giri S, Chung YC, Merchant A, Mihai G, Rajagopalan S, Raman SV, et al. T2 quantification for improved detection of myocardial edema. J Cardiovasc Magn Reson. 2009;11:56.

18. Giri S, Shah S, Xue H, Chung YC, Pennell ML, Guehring J, et al. Myocardia $\mathrm{T}(2)$ mapping with respiratory navigator and automatic nonrigid motion correction. Magn Reson Med. 2012;68(5):1570-8.
19. Blume U, Lockie T, Stehning C, Sinclair S, Uribe S, Razavi R, et al. Interleaved $T(1)$ and $T(2)$ relaxation time mapping for cardiac applications. J Magn Reson Imaging. 2009;29(2):480-7.

20. Kellman P, Hansen MS. T1-mapping in the heart: accuracy and precision. J Cardiovasc Magn Reson. 2014;16:2

21. Piechnik SK, Ferreira VM, Lewandowski AJ, Ntusi NA, Banerjee R, Holloway C, et al. Normal variation of magnetic resonance T1 relaxation times in the human population at 1.5 T using ShMOLLI. J Cardiovasc Magn Reson. 2013;15:13.

22. Cheng ASH, Pegg TJ, Karamitsos TD, Searle N, Jerosch-Herold M, Choudhury $\mathrm{RP}$, et al. Cardiovascular magnetic resonance perfusion imaging at 3-tesla for the detection of coronary artery disease. J Am Coll Cardiol. 2007:49(25):2440-9.

23. Roujol S, Foppa M, Weingartner S, Manning WJ, Nezafat R. Adaptive registration of varying contrast-weighted images for improved tissue characterization (ARCTIC): application to T1 mapping. Magn Reson Med. 2015;73(4):1469-82

24. Xue H, Greiser A, Zuehlsdorff S, Jolly MP, Guehring J, Arai AE, et al. Phase-sensitive inversion recovery for myocardial T1 mapping with motion correction and parametric fitting. Magn Reson Med. 2013;69(5):1408-20.

25. Xue H, Shah S, Greiser A, Guetter C, Littmann A, Jolly MP, et al. Motion correction for myocardial T1 mapping using image registration with synthetic image estimation. Magn Reson Med. 2012;67(6):1644-55.

26. Akçakaya M, Basha TA, Weingärtner S, Roujol S, Berg S, Nezafat R. Improved quantitative myocardial T2 mapping. Mag Reson Med 2014, In Press. doi:10.1002/mrm.25377.

27. Cornelius N, Kanade T. Adapting optical-flow to measure object motion in reflectance and X-ray image sequences. ACM SIGGRAPH Comput Graph. 1984;18:24-5.

28. Butler CR, Thompson R, Haykowsky M, Toma M, Paterson I. Cardiovascular magnetic resonance in the diagnosis of acute heart transplant rejection: a review. J Cardiovasc Magn Reson. 2009;11:7.

29. Roujol S, Benois-Pineau J, de Senneville BD, Quesson B, Ries M, Moonen C. Real time constrained motion estimation for ECG-gated cardiac MRI. 2010. IEEE. p 757-760.

30. Roujol S, Benois-Pineau J, de Senneville BD, Ries M, Quesson B, Moonen CT. Robust real-time-constrained estimation of respiratory motion for interventional MRI on mobile organs. IEEE Trans Inf Technol Biomed. 2012;16(3):365-74

31. Pratikakis I, Barillot C, Hellier P, Memin E. Robust multiscale deformable registration of 3D ultrasound images. International Journal of Image and Graphics. 2003;3(04):547-65.

32. de Senneville BD, Noe KO, Ries M, Pedersen M, Moonen CT, Sorensen T. An optimised multi-baseline approach for on-line MR-temperature monitoring on commodity graphics hardware. 2008. IEEE. p 1513-1516.

33. Ostergaard Noe K, De Senneville BD, Elstrom UV, Tanderup K, Sorensen TS. Acceleration and validation of optical flow based deformable registration for image-guided radiotherapy. Acta Oncol. 2008;47(7):1286-93.

34. Roujol S, Ries M, Quesson B, Moonen C, Denis de Senneville B. Real-time MR-thermometry and dosimetry for interventional guidance on abdominal organs. Magn Reson Med. 2010;63(4):1080-7.

35. Lourakis M. levmar: Levenberg-Marquardt nonlinear least squares algorithms in C/C++. www.ics.forth.gr/ lourakis/levmar/ 2004, Updated on November 29, 2011, Accessed on June 1, 2013

36. Dice LR. Measures of the amount of ecologic association between species. Ecology. 1945;26(3):297-302.

37. Cerqueira MD, Weissman NJ, Dilsizian V, Jacobs AK, Kaul S, Laskey WK, et al. Standardized myocardial segmentation and nomenclature for tomographic imaging of the heart. A statement for healthcare professionals from the Cardiac Imaging Committee of the Council on Clinical Cardiology of the American Heart Association Circulation. 2002;105(4):539-42.

38. Cheng C, Herfkens R, Taylor C. Inferior vena caval hemody- namics quantified in vivo at rest and during cycling exercise using magnetic resonance imaging. Am J Physiol Heart Circ Physiol. 2003;284(4):H1161-1167.

39. Ding H, Schär M, Zviman M, Halperin HR, Beinart R, Herzka DA. High-resolution quantitative 3D T2 mapping allows quantification of changes in edema after myocardial infarction. J Cardiovasc Magn Reson. 2013;15 Suppl 1:181.

40. Heeswijk RB, Piccini D, Feliciano H, Hullin R, Schwitter J, Stuber M. Self-navigated isotropic three-dimensional cardiac T2 mapping. Magn Reson Med. 2015;73(4):1549-54 